- Case Report

\title{
A Case Report of Pityriasis Lichenoides- Like Mycosis Fungoides in Children: A Challenging Diagnosis
}

\author{
Siti Noorfadhilah Mohd Amin', Rosediani Muhamad ${ }^{1}{ }^{*}$, Wan Noor Hasbee Wan Abdullah², Maryam Mohd Zulkifli', \\ Ikmal Hisyam Bakrin ${ }^{3}$, Teebah Tangam ${ }^{2}$
}

'Department of Family Medicine, School of Medical Sciences, Universiti Sains Malaysia, Kubang Kerian, Malaysia

${ }^{2}$ Department of Dermatology, Hospital Raja Perempuan Zainab II, Kota Bahru, Malaysia

${ }^{3}$ Department of Pathology, Universiti Putra Malaysia, Serdang, Malaysia

Primary cutaneous lymphomas are rare diseases among the general population, and even rarer in children. Mycosis fungoides (MF) is the most commonly diagnosed form in childhood. Several atypical clinical variants of MF have been reported, and pityriasis lichenoides-like MF (PL-like MF) is a recently described subtype. We report about a rare case of PL-like MF in an 11-year-old Malay boy with a 2-year history of multiple scaly erythematous papules associated with progressive and generalized hypopigmentation. This case report illustrates the significant dilemma in the diagnosis of the disease, particularly in the early stages, because its symptoms can mimic those of many common childhood inflammatory skin disorders. Later, the widespread hypopigmentation obscured the characteristic lesions, leading to misdiagnosis. Moreover, due to unfamiliarity of the disease, the diagnosis of PLlike MF was missed and delayed until only 2 years after the onset of the dermatosis. Therefore, primary care practitioners must have a high index of suspicion for this cutaneous neoplasm in children with persistent or worsening skin lesions, not responding to standard therapy, to ensure timely referral, diagnosis, and treatment.

Keywords: Child; Cutaneous T-Cell Lymphoma; Mycosis Fungoides; Pityriasis Lichenoides; Primary Cutaneous Lymphoma; Case Report

Received: March 3, 2020, Accepted: March 11, 2020

*Corresponding Author: Rosediani Muhamad https://orcid.org/0000-0002-8393-7899

Tel: +60-9-7676600, Fax: +60-9-7676611, E-mail: rosesyam@usm.my 


\section{INTRODUCTION}

Primary cutaneous lymphomas (PCL) are a heterogeneous group of skin malignancies of T-cell, B-cell, and, rarely, natural killer-cell origin. ${ }^{1,2)}$ They present primarily on the skin, without evidence of extracutaneous involvement. PCL with T-cell origin, known as cutaneous Tcell lymphomas (CTCL), constitute a vast majority of PCL cases, with mycosis fungoides (MF) being the most prevalent CTCL. ${ }^{1,3)} \mathrm{MF}$ is a low-grade non-Hodgkin lymphoma wherein the skin is infiltrated with neoplastic T-cells, thereby resulting in the formation of classical skin lesions, ranging from patches to plaques, and later transformation to cutaneous tumors. ${ }^{1-3)}$ However, patients can present with atypical cutaneous manifestations of MF such as hyperkeratotic, bullous, granulomatous, pustular, and verrucous lesions. ${ }^{3-5)}$ These diverse manifestations make the diagnosis of MF unquestionably challenging.

Recently, the distinct presentation of MF with pityriasis lichenoides (PL)-like lesions exhibiting recurrent crops of erythematous scaly papules has been reported. ${ }^{4,6-8)}$ These lesions can lead to widespread hypopigmentation in dark-skinned individuals, thereby masking the characteristic lesions and resulting in misdiagnosis and poor treatment. ${ }^{6)}$ This new subtype of MF is called pityriasis lichenoides-like MF (PL-like MF). Its clinical findings are similar to those of PL (benign reactive papulosquamous lesions), while its histopathological findings are similar to those of neoplastic MF. ${ }^{7)}$ PL-like MF is a very rare variant of MF, with only a few cases reported. The first case series was described by Ko et al. ${ }^{5)}$ in 2000, followed by few authors thereafter. ${ }^{4,5,7,8)}$ We thereby report a rare case of PL-like MF to raise awareness among primary care physicians regarding the importance of clinical suspicion of MF in children with persistent or worsening papular skin lesions, not responding to standard therapy, to ensure timely referral, diagnosis, and treatment.
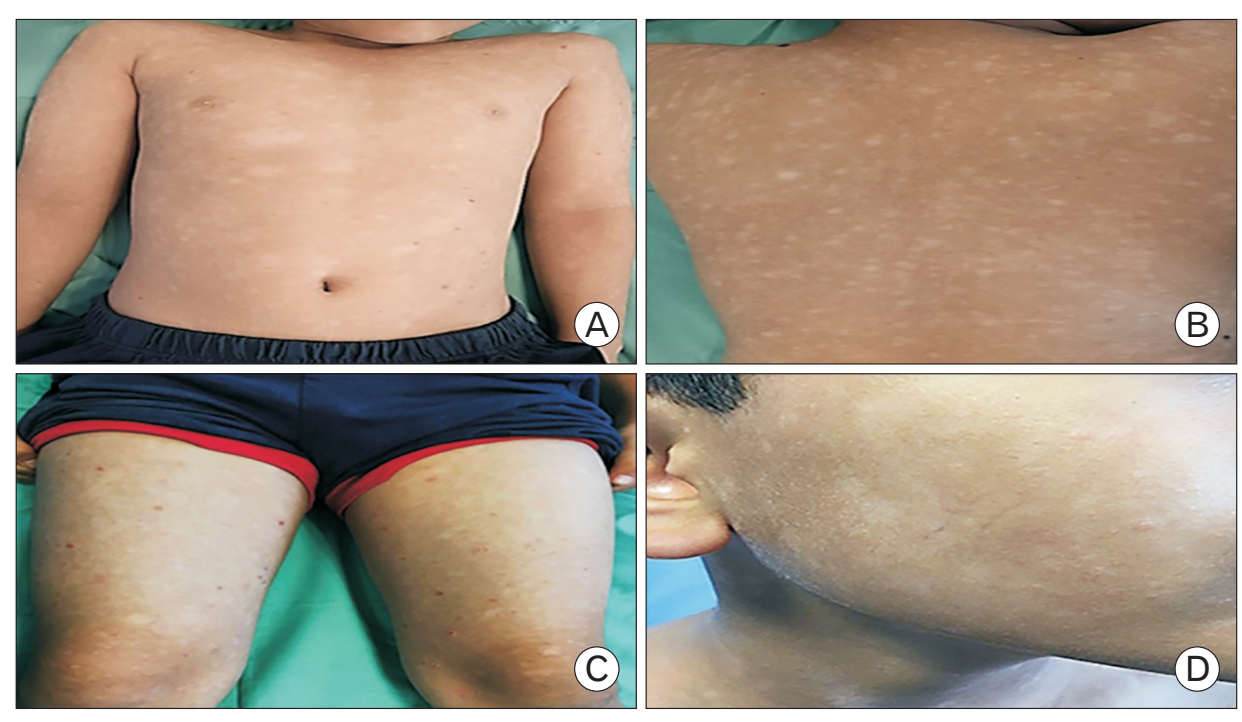

Figure 1. Generalized hypopigmented macules and patches over the trunk (A), back (B), limbs (C), and face (D), covering approximately $70 \%$ of the body surface area. Written informed consent for publication of this image was obtained from the patient. 
treatment, but his skin condition persisted and progressively worsened. The initial stage of pruritic erythematous papules had evoked the diagnosis of early varicella infection. Subsequently, he was treated for atopic dermatitis and pityriasis rosea with various topical emollients and steroids, with no significant improvement. Later, several diagnoses such as tinea corporis, pityriasis alba, pityriasis versicolor, and post inflammatory hypopigmentation were made for the progressive hypopigmented lesions. Antifungals and steroids were also prescribed, but none effectively cured the condition. Then, he was referred to a dermatologist for expert evaluation.

A 4.0-mm punch biopsy was taken from two sites: an erythematous papule and a hypopigmented macule. Histopathological studies on the erythematous papule revealed marked acanthosis and mild parakeratosis with prominent epidermotropism of the dermis. The lymphocytes were atypical in appearance, some of which appeared cerebriform. Pautrier's microabscess was occasionally seen. The papillary dermis had dense band-like lymphocytic infiltrates, with perivascular lymphocytic and histiocytic cells in the deep dermis (Figure 3). Immu-

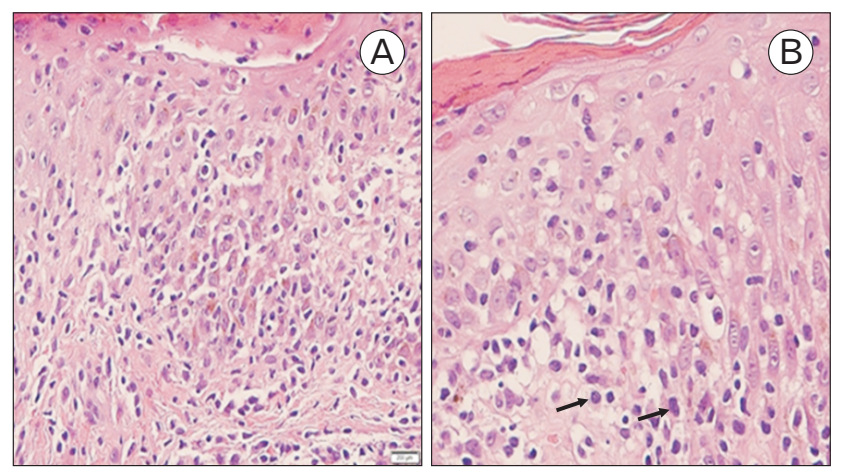

Figure 3. (A) Image of the epidermis showing marked epidermotropism of atypical lymphocytes with basilar tagging. (B) Some of the lymphocytes appeared cerebriform (arrows) (H\&E, $\times 400)$. nohistochemical staining demonstrated positivity for CD2+, CD3+, and CD5+ and negativity for CD30+ T-cells. CD8+ T-cell expression was more predominant than that of CD4+ T-cells (Figure 4). T-cell receptor gamma gene rearrangement analysis was not performed in this case. Otherwise, biopsy of the hypopigmented macules showed features of post-inflammatory hypopigmentation.

In summary, our patient had clinical presentation of pityriasis lichenoides-like lesions exhibiting recurrent crops of erythematous and scaly papules, which then resulted in prominent post-inflammatory hypopigmentation. Nevertheless, the histopathology combined with immunochemical findings were consistent with those of MF. Therefore, the diagnosis of PL-like MF was established. Treatment thrice weekly with narrowband ultraviolet B (NBUVB) phototherapy, along with topical betamethasone valerate cream $0.05 \%$ once daily, was commenced. Significant clinical improvement was observed after completion of 30 phototherapy sessions. A long-term periodic followup was planned in order to detect any early symptoms and signs of relapse. The patient provided written consent for the publication of clinical details and images.

\section{DISCUSSION}

Pityriasis lichenniodes (PL) and MF are traditionally regarded as two different entities, representing the most common benign and malignant lymphoproliferative disorders in children, respectively. ${ }^{9,10)} \mathrm{PL}$ is characterized by reactive cutaneous eruptions of erythematous scaly papules, while MF has a wide range of clinical presentations depending on its variants. Histopathologic findings of PL include necrotic keratinocytes, spongiosis, erythrocyte extravasation, and perivascular cell infiltration. ${ }^{4,7)}$ In MF, the remarkable features are Pautrier's microabscess, epidermotropism, haloed lymphocytes, and coarse collagen bundles in the papillary dermis. ${ }^{4,5}$ Recent evidence suggested a relationship between PL and MF, categorized into three groups: PL with T-
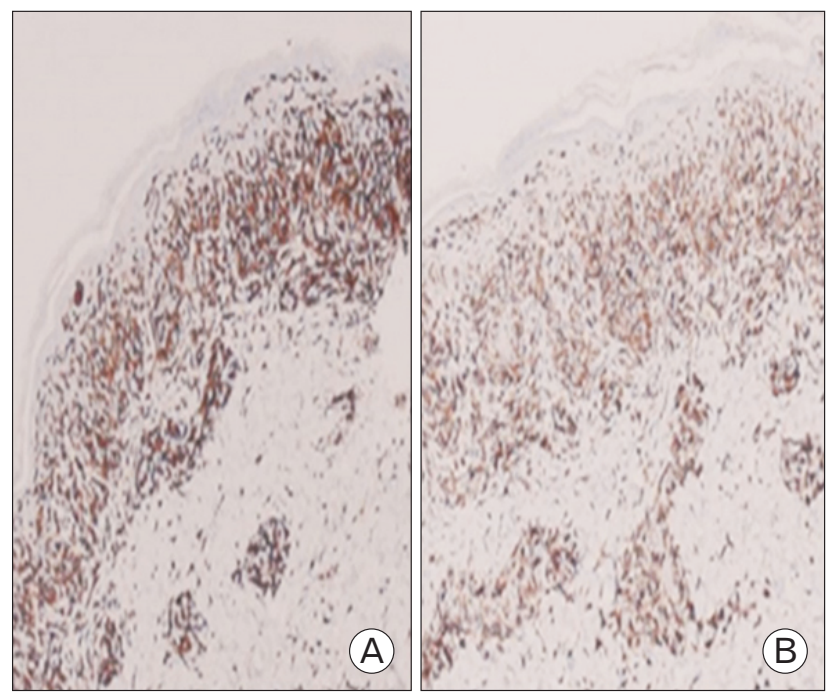

Figure 4. Immunohistochemical images demonstrating positivity for $\mathrm{CD} 3(\mathrm{~A}), \mathrm{CD} 8(\mathrm{~B})$, and Granzyme B (C) (immunohistochemical stain, $\times 200$ ). 
cell infiltration, PL evolving to MF, and PL-like MF.-10) PL-like MF is a rare subtype of MF, described as causing similar benign skin lesions as those of PL but having histopathological findings as those of malignant MF, as illustrated in our case.

The clinical pattern of the PL-like lesion, which is scaly erythematous papules, can be easily mistaken for other more common benign skin conditions. In our case, the patient first arrived at the clinic after having pruritic erythematous papule eruption for 2 days, and the provisional diagnosis of varicella infection was made. However, the absence of vesicles, slow progression, and lack of prodromal symptoms did not further support this diagnosis. Other diagnoses made by different primary care physicians were atopic dermatitis and pityriasis rosea in view of the papulosquamous rashes. Therefore, the patient was treated with various topical emollients and steroids but showed no significant improvement.

Over several weeks, some erythematous lesions slowly faded, and hypopigmented macules and patches started to appear. Primary care physicians face significant dilemma in the diagnosis of hypopigmented skin lesions because the differential blood tests are vast. When the patient presented with fewer hypopigmented macules, the diagnosis was most likely considered as pityriasis alba. Other diagnoses made were superficial fungal infection including tinea corporis, pityriasis versicolor, and post-inflammatory hypopigmentation. Fungal infection can be easily excluded by microscopic examination of skin scrapings in potassium hydroxide solution, but unfortunately, none of the primary care physicians performed the test on this patient. He was treated clinically with topical and oral antifungal medications for a few months, but the hypopigmentation worsened, affecting almost his entire body. Differential diagnoses of vitiligo and leprosy must also be considered in hypopigmentation, but these were very unlikely in this case.

In dark-skinned individuals, the risk for post-inflammatory hypopigmentation after any cutaneous inflammation significantly increases. ${ }^{6}$ ) In this case, widespread hypopigmentation after the resolution of erythematous papules occurred, covering almost his entire body. Extensive hypopigmentation also caused primary care physicians to disregard the residual erythematous lesions, hence only formulating diagnoses for hypopigmentation. A detailed history including the progression of skin lesions, response to previous treatments, and a thorough physical examination must be performed to avoid misdiagnosis and improper treatment. If the skin lesion improves minimally, persists, or worsens, then primary care physicians should refer the case for expert evaluation to avoid undue delay in making a definitive diagnosis. A skin biopsy is often helpful in diagnosing suspicious skin lesions.

In conclusion, PL-like MF is an uncommon and challenging diagnosis not only for primary care physicians but also for dermatologists and pathologists. It is a rare malignant cutaneous disease with overlapping features of benign inflammatory disorders and conflicting clinicopathological findings. This condition should also be taken into consideration during suspected diagnosis in children with persistent or worsening skin lesions, not responding to standard topical treat- ment. Appropriate referral for expert evaluation and skin biopsy will ultimately lead to timely diagnosis and better outcome. The first-line treatment for children with PL-like MF is phototherapy with NBUVB, and a favorable response can be anticipated. ${ }^{5,8)}$

\section{CONFLICT OF INTEREST}

No potential conflict of interest relevant to this article was reported.

\section{ACKNOWLEDGMENTS}

We would like to acknowledge the contributions made by Department of Dermatology (Hospital Raja Perempuan Zainab II) and Department of Pathology (Hospital Raja Perempuan Zainab II and Hospital Serdang) for their assistance with the images, and the patient for giving his consent for this case report.

\section{ORCID}

Siti Noorfadhilah Mohd Amin: https://orcid.org/0000-0002-1877-2901 Rosediani Muhamad: https://orcid.org/0000-0002-8393-7899 Wan Noor Hasbee Wan Abdullah: https://orcid.org/0000-0001-5610-7833 Maryam Mohd Zulkifli: https://orcid.org/0000-0002-2413-5263

Ikmal Hisyam Bakrin: https://orcid.org/0000-0002-9681-4118

Teebah Tangam: https://orcid.org/0000-0002-3077-132X

\section{REFERENCES}

1. Fitzpatrick TB, Freedberg IM. Fitzpatrick's dermatology in general medicine. 8th ed. New York (NY): MacGraw-Hill; 2008.

2. Jawed SI, Myskowski PL, Horwitz S, Moskowitz A, Querfeld C. Primary cutaneous T-cell lymphoma (mycosis fungoides and Sezary syndrome): part I: diagnosis: clinical and histopathologic features and new molecular and biologic markers. J Am Acad Dermatol 2014;70:205.

3. Ahn CS, ALSayyah A, Sangueza OP. Mycosis fungoides: an updated review of clinicopathologic variants. Am J Dermatopathol 2014;36:933-48.

4. Jang MS, Kang DY, Park JB, Kim JH, Park KA, Rim H, et al. Pityriasis lichenoides-like mycosis fungoides: clinical and histologic features and response to phototherapy. Ann Dermatol 2016;28:540-7.

5. Ko JW, Seong JY, Suh KS, Kim ST. Pityriasis lichenoides-like mycosis fungoides in children. Br J Dermatol 2000;142:347-52.

6. Lane TN, Parker SS. Pityriasis lichenoides chronica in black patients. Cutis 2010;85:125-9.

7. De Unamuno Bustos B, Ferriols AP, Sanchez RB, Rabasco AG, Vela CG, Piris MA, et al. Adult pityriasis lichenoides-like mycosis fungoides: a clinical variant of mycosis fungoides. Int J Dermatol 2014;53:1331-8.

8. Lin TL, Chen YJ, Weng YC, Yang CS, Juan IK. Pityriasis lichenoid-like mycosis fungoides in a 9-year-old boy: a case report. Acta Dermatovenerol Croat 2019;27:37-9.

9. Zaaroura H, Sahar D, Bick T, Bergman R. Relationship between pityriasis lichenoides and mycosis fungoides: a clinicopathological, immunohistochemical, and molecular study. Am J Dermatopathol 2018;40: 409-15. 
10. Boos MD, Samimi SS, Rook AH, Yan AC, Kim EJ. Pityriasis lichenoides and cutaneous T cell lymphoma: an update on the diagnosis and management of the most common benign and malignant cutaneous lymphoproliferative diseases in children. Curr Dermatol Rep 2013;2: 203-11. 